Europhys. Lett., 19 (3), pp. 183-187 (1992)

\title{
The Fractal Dimension of Iso-Vorticity Structures in 3-Dimensional Turbulence.
}

\author{
I. Procaccia $\left(^{*}\right)(* *)$, A. Brandenburg $(* *)$, M. H. Jensen $(* *)$ and A. Vincent $(* * *)$

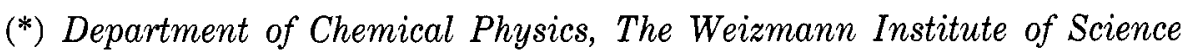 \\ Rehovot 76100, Israel \\ (**) Nordita - Blegdamsvej 17, DK-2100 Copenhagen Ø, Denmark \\ (***) CERFACS - 42 Avenue Coriolis, F-31057 Toulouse, France
}

(received 11 November 1991; accepted in final form 27 April 1992)

PACS. $47.25 \mathrm{C}$ - Isotropic turbulence.

\begin{abstract}
The fractal dimension of iso-vorticity surfaces is estimated from a 3-dimensional simulation of homogeneous turbulence at moderate Reynolds numbers, performed by Vincent and Meneguzzi. The results are found to be compatible with a recently proposed theory which predicts a crossover from a 2-dimensional geometry at small scales to a fractal geometry at larger scales, with a dimension $D=2.5+\zeta / 2$, with $\zeta$ being the exponent characterizing the scaling of velocity differences.
\end{abstract}

One promising venue for the search of universal aspects in fluid turbulence is the study of universal geometrical structures. Mandelbrot [1] has suggested that such universal structures may be fractal, exhibiting the self-similarity on a range of scales which is at the heart of the scaling properties of turbulence. Some encouraging progress in identifying candidates for universal fractal structures has been achieved experimentally [2], but only little help was provided from theoretical studies; most of these were completely phenomenological $[3,4]$, and theories based directly on the equations of fluid mechanics failed to provide insight towards the existence and the characterization of fractal structures in turbulence.

Recently, an approach based on fluid mechanics and on concepts of geometric measure theory was proposed [5], and some progress in calculating the dimensions of level sets of both passive scalars [6,7] and vorticity magnitude [8] were reported. The results concerning the properties of passive scalars were compared directly with experiments [7]. In this letter we offer a comparison of the predictions on the geometries of iso-vorticity surfaces to the results of numerical simulations of homogeneous turbulence at moderate Reynolds numbers [9].

We begin with a short summary of the theoretical predictions. Consider the Navier-Stokes equation

$$
\partial \boldsymbol{u} / \partial t-v \nabla^{2} \boldsymbol{u}+\boldsymbol{u} \cdot \nabla \boldsymbol{u}+\nabla p=\boldsymbol{F},
$$

where $v, p$ and $\boldsymbol{F}$ are the viscosity, pressure and external forcing, respectively. Equation (1) is considered in three spatial dimensions, in a volume $V$ (of linear scale $L$ ) and is 
supplemented with the incompressibility condition $\nabla \cdot \boldsymbol{u}=0$. The vorticity $\boldsymbol{w}$ is the curl of $\boldsymbol{u}$, $\boldsymbol{w}=\nabla \times \boldsymbol{u}$, and its magnitude $|\boldsymbol{w}|$ is denoted by $w$. To derive an equation of motion for $w$ one takes the curl of eq. (1), multiplies the result by $\boldsymbol{w}$, and divides by $w$. The resulting equation reads (for $w \neq 0$ )

$$
\partial w / \partial t+\boldsymbol{u} \cdot \nabla w-v \nabla^{2} w=\left(\alpha-v|\nabla \xi|^{2}\right) w
$$

where

$$
\alpha=(\boldsymbol{w} \cdot \nabla \boldsymbol{u}) \cdot \boldsymbol{w} / w^{2} ; \quad \xi=\boldsymbol{w} / w
$$

The term on the r.h.s. of eq. (2) is the only difference between the dynamics of $w$ and the dynamics of a passive scalar like the temperature, $\partial T / \partial t+\boldsymbol{u} \cdot \nabla T-k \nabla^{2} T=0$. In the regions of space where the coefficient $f(x, t) \equiv\left(\alpha-v|\nabla \xi|^{2}\right)$ is positive, $w$ grows rapidly; in regions where it is negative, $w$ decreases rapidly. It is this term that must be responsible for the rapid concentration of the field $w$, if there is one. The geometrical objects that are the subject of this letter are the regions in 3-space in which the magnitude of the vorticity $w$ has a constant value at some instance of time. The theoretical question is what are the generic geometrical properties of these iso-vorticity sets, and what is their fractal dimension if they turn out to be fractal.

The technique [5-8] to estimate the dimension of iso- $w$ surfaces rested on a calculation of the area of a piece of this surface contained in a ball $B$ of diameter $r$. By changing the diameter $r$ one obtained an expression for the $r$-dependence of this area. For a smooth 2-dimensional surface this area should scale like $r^{2}$. For a wrinkled, fractal surface the exponent is larger than 2. The estimate of this exponent, denoted as $D$, was the goal of ref. [8]. In order to achieve a generic, rather than an atypical value of the dimension which might result from fluctuations, an average over a short interval of time (of the order of a turn over time), and an average over a band of $w$ values has been achieved. The calculation takes into account explicitly the fast increase in $w$ in regions where the r.h.s. of eq. (2) is positive; see ref. [8] for details. The final result of the calculation can be written in the form

$$
r^{2 D} \leqslant r^{4}\left[c_{1}+\frac{c_{2} r \delta u_{r}}{v}\right]
$$

where $c_{1}, c_{2}$ are nondimensional constants, and the quantity $\delta u_{r}$ is defined as

$$
\delta u_{r}=\frac{1}{\delta t} \frac{3}{4 \pi r^{3}} \int_{t_{0}}^{t_{0}+\delta t} \mathrm{~d} t \int_{B}\left|\boldsymbol{u}(\boldsymbol{x}, t)-\boldsymbol{u}_{0}\right| \mathrm{d}^{3} x,
$$

where $\delta t$ is the interval of time over which the averaging is done. $\boldsymbol{u}_{0}$ is the average velocity in the ball $B$.

The 1.h.s. of eq. (4) represents an average value of the square of the area of the pieces of the iso- $w$ sets contained in a ball of radius $r$, averaged over a short interval of time. For sufficiently small $r$, or sufficiently large viscosity, the first term $c_{1} r^{4}$ dominates, and the iso- $w$ surfaces are smooth. The entire $w$ field is then nicely foliated by the iso- $w$ surfaces, and there is nothing irregular about the geometry of the vorticity. However, when the viscosity $v$ goes down (i.e. the Reynolds number $R e=U L / v$ goes up, with $U$ being the typical velocity at the integral scale $L$ ), the other term on the r.h.s. of (4) takes over, and the geometry of the iso- $w$ surfaces changes qualitatively. The result embodied in (4) is based on a rigorous bound on the area of the level sets. In order to proceed, one can introduce a scaling assumption, i.e. that 
$\delta u_{r}$ scales with $r$,

$$
\delta u_{r} \sim U(r / L)^{\zeta} \sim\left|\boldsymbol{u}_{0}\right|(r / L)^{\zeta},
$$

with $\zeta$ being $1 / 3$ in the Kolmogorov theory [10]. With this assumption in mind one can see that the second term in eq. (4) contributes an overall $r^{5+\zeta}$. The second term begins to dominate the first on length scales $r \geqslant \lambda^{*}$, where $\lambda^{*}$ is defined by

$$
\frac{\hat{\lambda}^{*} \delta u_{\lambda^{*}}}{v}=\frac{c_{1}}{c_{2}}
$$

We notice that eq. (7) is nothing but a statement about the local Reynolds number, calculated on the scale $r=\lambda^{*}$. When this scale-dependent Reynolds number exceeds the value $c_{1} / c_{2}$, the iso- $w$ surface will not appear smooth. It has been found before [7] that the crossover between smooth and fractal behaviour occurs at a scale $\lambda^{*}$ which is about an order of magnitude larger than the standard Kolmogorov dissipative scale $\eta$. ( $\lambda^{*}$ scales with the Reynolds number exactly like $\eta$; the reason for the difference in order of magnitude is that $\eta$ is computed from an equation like (7) but with 1 on the r.h.s. Typically $c_{1} / c_{2}$ differs significantly from unity.) Thus, we expect that for scales $r$ larger than $\lambda^{*}$ the iso-vorticity structures would appear fractal with a dimension $D \leqslant 2.5+\zeta / 2$. Although the rigorous result implies an inequality, it is possible that the bound is saturated; if it does, this has interesting implications on the general theory of turbulence [8]. Also, the theory cannot exclude the possibility that the scaling exponent $D$ in eq. (4) depends on $r$. One of the aims of the present study is to assess these issues further with the help of numerical simulations.

To test these predictions we have analysed the data obtained by a numerical simulation of homogeneous turbulence [9]. The resolution of this simulation is $240^{3}$ Fourier components and periodic boundary conditions are assumed in all 3 directions. The flow is incompressible and forced with constant amplitude on the lowest wave numbers. The Reynolds number is around 1000 , and the Taylor-microscale Reynolds number $R e_{\lambda}$ is approximately 150 . The Kolmogorov dissipative scale $\eta$ is about $2 \div 4$ mesh sizes, whereas the Taylor microscale is about $10 \div 20$ mesh sizes.

The fully developed flow shows a large number of vortex tubes with a typical thickness of a few $\eta$, extending through a substantial fraction of the system size (see fig. 13 in ref. [9]). We are interested in level sets of $w$. These are obtained by considering the sets of mesh points for which this quantity lies in given windows, $w_{1} \leqslant w \leqslant w_{2}$. The typical 3-dimensional appearance of these sets can be seen in fig. 1, which portrays the set for a particular window which is frequently used below, i.e. $50 \leqslant w \leqslant 51$. It appears that on the larger scales this set is neither 2-dimensional nor space filling, and it is possible that it indeed possesses a fractal geometry.

To examine this apparent fractal geometry we determine the dimension of the set using the Grassberger-Procaccia correlation integral [11]. As is well known, in general this method furnishes the correlation dimension [11] rather than the Hausdorff dimension, but for an iso-level set there is no difference between the two. (Only singularities in the measure can lead to a difference, and by definition the measure is constant on a level set.) Denoting the locations of the mesh points in the set by $\boldsymbol{x}_{i}$, we calculate the correlation integral [11]:

$$
C(r)=\sum_{i \neq j=1}^{N} \Theta\left(r-\left|\boldsymbol{x}_{i}-\boldsymbol{x}_{j}\right|\right),
$$

where $\Theta(x)$ is the Heaviside step function. The correlation integral scales like $r^{D}$, where $D$ is the dimension of the set $\left\{\boldsymbol{x}_{i}\right\}$. In fig. 2 we show a $\log -\log$ plot of $C(r) v s . r$ for a typical window of $w$, which is the window used in fig. 1 . We have averaged the correlation integral over 26 


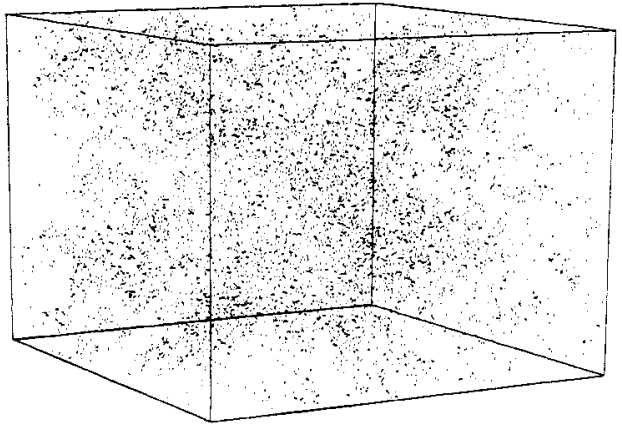

Fig. 1.

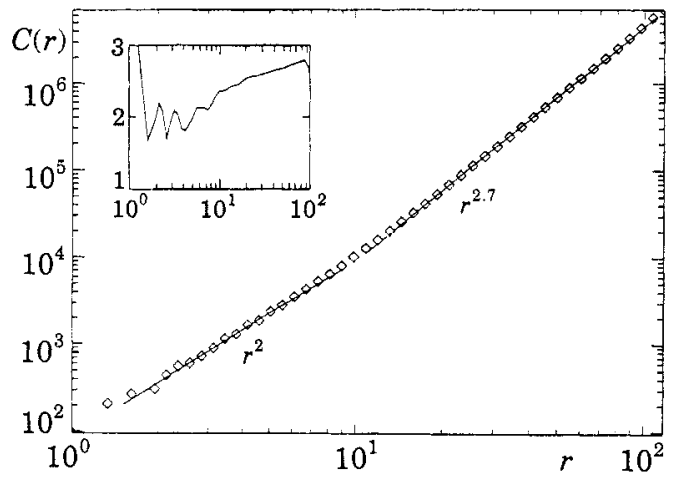

Fig. 2.

Fig. 1. - Visualization of the set of pixels for which the pointwise magnitude of the vorticity lies in the window $50<w<51$, for \# 226 .

Fig. 2. - The correlation integral $C(r)$, averaged over 26 snapshots, $v s . r$ for the window shown in fig. 1. The inset shows the slope $d \ln C(r) / d \ln r$ vs. $r$.

equally spaced snapshots between \# 151 and \#401, covering approximately 50 turnover times. The duration between subsequently stored snapshots in this data set is about 2 turnover times.

Examining the results one concludes that $C(r)$ typically shows a crossover between two different scaling laws with an exponent close to 2 for $r<10 \eta$ (which is $\lambda^{*}$, or approximately one Taylor microscale), and an exponent of about 2.7 for $r>10 \eta$. The shape of $C(r)$ changes somewhat with time. This can be seen in fig. 3, where we plot $d \ln C(r) / d \ln r$ for the 26 snapshots between \# 151 and \#401. If we accepted the value of $D \simeq 2.7$ we would deduce a value for $\zeta$ which is about 0.4 . One should also notice the tendency towards space filling at the high end of the scaling range. It is difficult to determine at this point whether this is an artifact or a real worry about the existence of scaling behaviour.

Obviously, in qualitative terms, the findings reported here are in accord with the theoretical expectations. To gain a quantitative comparison we rewrite eq. (4) in the form

$$
r^{2 D}-r^{4}\left[c_{1}+c_{2} \operatorname{Re}\left(\frac{r}{L}\right)^{1+\zeta}+\ldots\right] .
$$

Assuming that the 1.h.s. of eq. (9) is proportional to $C(r)^{2}$ we can use our result to fit $\zeta$ and $c_{2} / c_{1}$ simultaneously. In practice we plot

$$
g=\frac{\mathrm{d} \ln \left[C(r)^{2} / r^{4}-a\right]}{\mathrm{d} \ln r}
$$

and vary the value of $a$ in order to find optimal scaling for $r>\lambda^{*}$ over a reasonable range. The value of $g$ for which this is the case corresponds then to $1+\zeta$. From this fit to $\ln \left[C(r)^{2} / r^{4}-a\right]$ vs. $\ln r$, we can obtain the ratio $c_{2} / c_{1}$. The procedure is demonstrated in fig. 4. This way of fitting produces a somewhat larger estimate for $\zeta$, and shows that the uncertainty is around $(20 \div 30) \%$. Anyway, we can conclude that this data set is consistent with a value of $\zeta$ which is significantly different from $1 / 3$, which is the Kolmogorov value. Note that a direct estimate of $\zeta$ in ref. [9] gives a value consistent with 0.4 .

In summary, we presented some numerical evidence for the existence of a crossover 


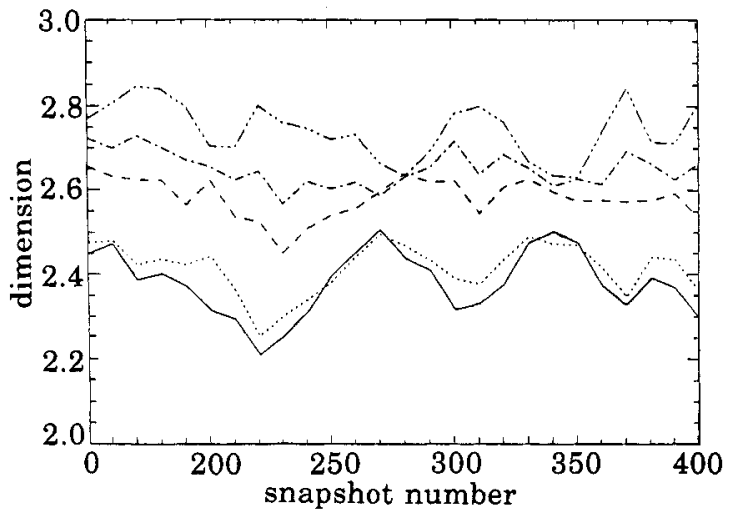

Fig. 3.

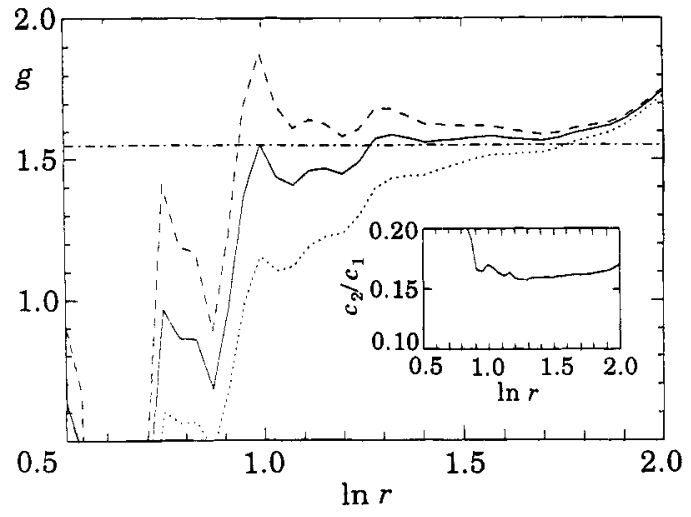

Fig. 4.

Fig. 3. - Variation of the local slope $\mathrm{d} \ln C(r) / \mathrm{d} \ln r$ between snapshots \# 151 and \# 401 for different values of $r(10(-), 16(\cdots), 26(---), 41(\cdot-\cdot-), 66(-\cdots-)$ mesh sizes).

Fig. 4. - The fit of $\ln \left[C^{2} / r^{4}-a\right] v s$. $\ln r$ for viscous values of $a$. The slope $g$ gives the ratio $c_{2} / c_{1}$ shown in the inset.

between smooth and fractal behaviour of the level sets of the vorticity magnitude. Due to the modest scaling range in these simulations it is impossible to state with confidence that the fractal behaviour is clear-cut. However, if we fit an exponent $D$ in the scaling range, we find a value that is in encouraging agreement with the theoretical predictions. Obviously, further experimental and simulational tests of these issues are called for before final conclusions can be reached.

IP acknowledges the partial support of the German-Israeli Foundation and the US-Israel Binational Fund.

\section{REFERENCES}

[1] Mandelbrot B. B., J. Fluid Mech., 62 (1974) 331; see also Temam R. (Editor), Turbulence and Navier-Stokes Equations, in Lecture Notes in Mathematics, Vol. 565 (Springer) 1976, p. 121.

[2] Sreenivasan K. R., Ann. Rev. Fluid Mech., 23 (1991) 539 and references therein.

[3] Frisch U., Sulem P. and Nelkin M., J. Fluid Mech., 63 (1978) 305.

[4] Benzi R., Paladin G., Parisi G. and Vulpiani A., J. Phys. A, 17 (1984) 3521.

[5] Constantin P., Commun. Math. Phys., 129 (1990) 241.

[6] Procaccia I., Ching E. S. C., Constantin P., Kadanoff L. P., Libchaber A. and Wu X.-Z., Phys. Rev. A, in press.

[7] Constantin P., Procaccia I. and Sreenivasan K. R., Phys. Rev. Lett., 67 (1991) 1739.

[8] Constantin P. and Procaccia I., Phys. Rev. A, in press.

[9] Vincent A. and Meneguzzi M., J. Fluid Mech., 225 (1991) 1.

[10] Kolmogorov A. N., Dokl. Akad. Nauk SSSR, 30 (1991) 299.

[11] Grassberger P. and Procaccia I., Phys. Rev. Lett., 50 (1983) 346. 